REGARDS

SUR L'ECONOMIE ALLEMANDE

BULLETIN ECONOMIQUE DUCIRAC

\section{Regards sur l'économie allemande}

Bulletin économique du CIRAC

$78 \mid 2006$

Varia

\title{
Vieillissement démographique : entre déni et dramatisation
}

\section{Wolfgang Schroeder et Arijana Neumann}

Traducteur : Isabelle Bourgeois

\section{OpenEdition \\ Journals}

Édition électronique

URL : http://journals.openedition.org/rea/752

DOI : $10.4000 /$ rea. 752

ISBN : 978-2-8218-0852-2

ISSN : 1965-0787

Éditeur

CIRAC

Édition imprimée

Date de publication : 1 octobre 2006

Pagination : 23-28

ISSN : 1156-8992

Référence électronique

Wolfgang Schroeder et Arijana Neumann, « Vieillissement démographique : entre déni et

dramatisation », Regards sur l'économie allemande [En ligne], 78 | octobre 2006, document 3, mis en ligne le 01 octobre 2008, consulté le 20 avril 2019. URL : http://journals.openedition.org/rea/752 ; DOI : 10.4000/rea.752 


\title{
Vieillissement démographique : entre déni et dramatisation
}

\author{
Wolfgang Schroeder et Arijana Neumann
}

En Allemagne, le débat de fond sur le vieillissement démographique et la baisse de la population n'a été lancé que voilà peu. Longtemps en effet, ces questions étaient traitées avec la plus grande prudence car considérées comme appartenant au fonds idéologique national-socialiste, ce qui interdisait de les affronter. Mais depuis le milieu des années 1990, la situation a radicalement changé : l'ampleur et l'effet des mutations démographiques font l'objet d'un vaste débat contradictoire au sein du monde politique et dans l'ensemble de la société. Comme souvent dans ces cas, deux camps extrêmes s'affrontent : d'un côté, ceux qui dramatisent les mutations et prédisent le déclin de l'Allemagne ; de l'autre, ceux qui dénient tout caractère préoccupant aux évolutions. Mais il existe aussi les tenants d'une ligne médiane, que nous représentons ici, et qui estiment que, si le vieillissement est un défi pour la société, il peut et doit être maîtrisé grâce à une politique collective d'accompagnement.

Longtemps prévalait outre-Rhin cette sentence de Konrad Adenauer: "Les Allemands n'ont pas besoin d'aide pour faire des enfants! ». Mais après les années du baby-boom (1955-65), et avec la diffusion de la pilule contraceptive, la natalité a amorcé sa chute. Sans nul doute, l'une des raisons en fut l'individualisation. A cette tendance affectant toutes les sociétés européennes s'ajouta en Allemagne une politique familiale qui, en se concentrant presque exclusivement sur le développement des transferts sociaux au détriment de celui des infrastructures d'accueil, interdit de concilier vies familiale et professionnelle.

Dans toute l'Europe, la population tend à vieillir sous l'effet de deux tendances : l'espérance de vie s'allonge, et le taux de natalité stagne à un niveau faible (Mai, 2003). Cela vaut aussi pour l'Allemagne où on observe donc non seulement le vieillissement de la population, mais aussi sa diminution : de 82 millions aujourd'hui, le nombre d'habitants devrait tomber à environ 75 millions en 2050 (Destatis, 2003). La structure de la population s'y caractérise d'abord par le fait que, depuis les années 1970, le taux de reproduction est inférieur au niveau requis pour assurer le renouvellement des générations. Avec 1,3 enfant environ par femme (Micheel, 2005), le taux de fécondité est très faible, même en comparaison internationale. Les femmes sont de plus en plus âgées à la naissance de leur premier enfant (elles ont aujourd'hui 29 ans, contre 22 ans en 1964), ce qui contribue à la baisse du nombre d'enfants par femme. Et un nombre croissant d'entre elles reste sans enfant (Mai, 2003) : alors que les femmes nées dans les années 1940 n'étaient que $10 \%$ à $15 \%$ dans ce cas, cette part est passée à $30 \%$ pour les femmes nées en 1960 . Quant à l'espérance de vie des femmes, elle atteint aujourd'hui 80,8 ans, celle des hommes, 74,8 ans ; ce niveau est élevé, quoique légèrement inférieur à la moyenne européenne.

La chute de la natalité a été rapide après 1965. Et la génération des nombreux baby-boomers générera sous peu un véritable papy-boom. A moyen terme, la relation entre les générations va ainsi s'inverser en Allemagne: la part des moins de 20 ans par rapport aux 20-59 ans (taux de jeunesse) y sera faible, alors que celle des plus de 60 ans par rapport aux 20-59 ans (taux de vieillesse) sera, elle, très élevée. Mais les débats sur le vieillissement retiennent

Une politique familiale qui a négligé les structures d'accueil

Vieillissement et diminution de la population

Bientôt inversion du rapport entre les générations 
une autre donnée qui mêle ces deux taux liés à l'âge au critère de l'activité : c'est le taux de dépendance. II s'agit de la relation entre les actifs (les 20 à 59 ans) et les inactifs (les moins de 20 ans et les plus de 60 ans).

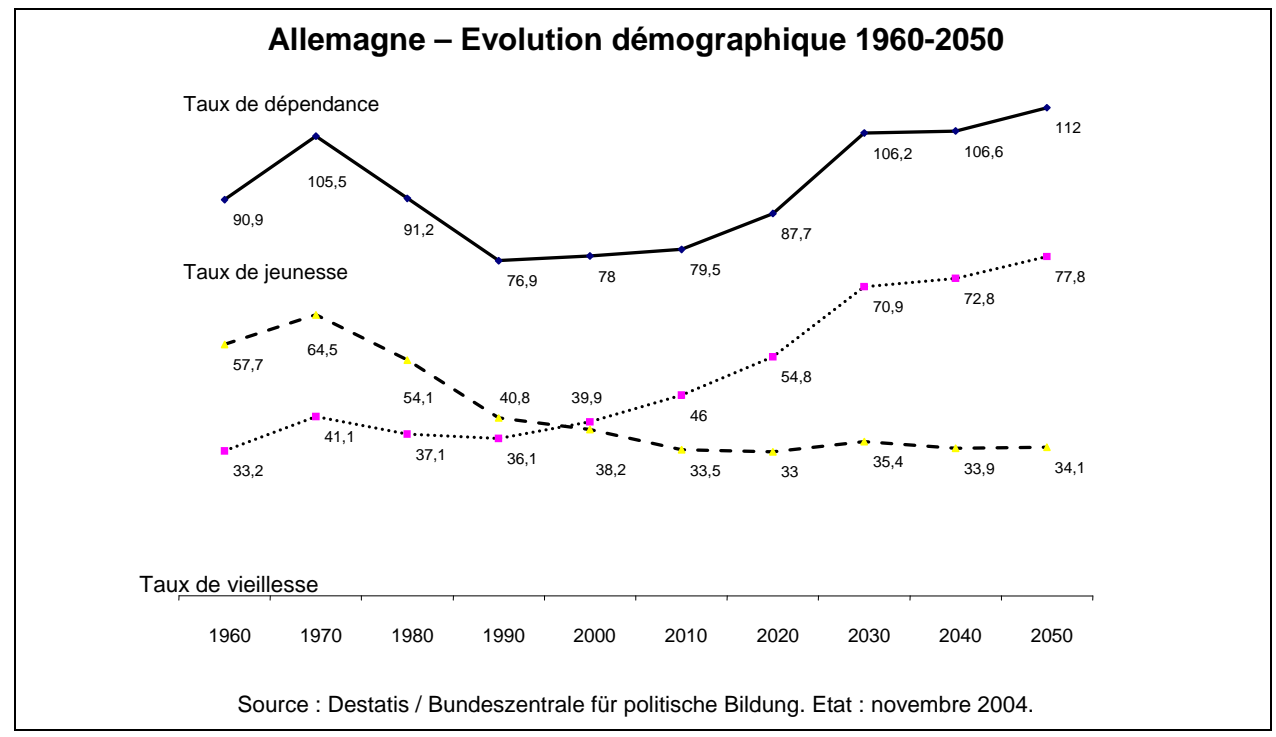

Or seule la composition du taux de dépendance est significative. L'Irlande, par exemple, aura en 2050 un taux de dépendance supérieur à l'Allemagne - sans qu'on puisse pour autant en conclure à un vieillissement caractérisé de sa population, puisque la part des jeunes y sera très forte. On peut faire la même remarque à propos de la France. A l'inverse, l'évolution de la démographie de l'Espagne et de l'Italie se révèle plus problématique encore que celle de l'Allemagne, dans la mesure où on y observe un taux de vieillesse très élevé.

\begin{tabular}{|l|cc|cc|cc|}
\hline & \multicolumn{2}{|c|}{ Taux de jeunesse } & \multicolumn{2}{c|}{ Taux de vieillesse } & \multicolumn{2}{c|}{ Taux de dépendance } \\
& $\mathbf{2 0 0 0}$ & $\mathbf{2 0 5 0}$ & $\mathbf{2 0 0 0}$ & $\mathbf{2 0 5 0}$ & $\mathbf{2 0 0 0}$ & $\mathbf{2 0 5 0}$ \\
UE 15 & 41,7 & 41,2 & 38,8 & 71,5 & 80,5 & 112,8 \\
Allemagne & $\mathbf{3 8 , 6}$ & $\mathbf{3 7 , 5}$ & $\mathbf{4 0 , 4}$ & $\mathbf{7 2 , 4}$ & $\mathbf{7 9 , 0}$ & $\mathbf{1 0 9 , 8}$ \\
Autriche & 40,6 & 39,4 & 35,4 & 67,3 & 76,1 & 106,6 \\
Belgique & 43,4 & 44,2 & 40,1 & 66,5 & 83,5 & 110,6 \\
Danemark & 42,0 & 43,3 & 34,7 & 57,4 & 76,7 & 100,8 \\
Espagne & 38,7 & 38,9 & 38,0 & 82,5 & 76,7 & 121,4 \\
Finlande & 44,8 & 43,9 & 35,4 & 64,7 & 80,3 & 108,7 \\
France & 46,9 & 44,8 & 37,9 & 70,8 & 84,7 & 115,7 \\
Grèce & 41,1 & 43,0 & 41,9 & 71,4 & 83,1 & 114,4 \\
Irlande & 58,0 & 45,1 & 29,1 & 68,9 & 87,0 & 114,1 \\
Italie & 35,6 & 37,7 & 42,3 & 81,5 & 77,9 & 119,2 \\
Luxembourg & 44,3 & 43,9 & 34,3 & 57,8 & 78,7 & 101,6 \\
Pays Bas & 42,4 & 44,0 & 31,7 & 62,3 & 74,1 & 106,2 \\
Portugal & 42,4 & 42,8 & 36,9 & 65,5 & 79,2 & 108,3 \\
Royaume Uni & 46,8 & 43,4 & 37,8 & 66,7 & 84,6 & 110,1 \\
Suède & 46,3 & 45,9 & 41,1 & 59,1 & 87,4 & 105,0 \\
\hline
\end{tabular}

Source des données : Mai, 2003. Taux de jeunesse (Jugendquotient) $:<20$ ans / 20-59 ans ; Taux de vieillesse (Altenquotient) : > 60 ans / 20-29 ans ; Taux de dépendance (Gesamtquotient) : actifs (20-59 ans) / inactifs (0-19 ans et $>60$ ans).

\section{Le débat politique et scientifique sur la démographie}

3 approches dans le débat

Ceux qui « dramatisent »
Dans le débat allemand sur le vieillissement démographique, qu'il soit scientifique ou politique, on peut distinguer grosso modo trois types d'approches.

La première rassemble les tenants de la "dramatisation ». Ils interprètent les mutations démographiques comme une menace pesant sur l'Etat Providence tel que nous le connaissons. Nous devons donc en faire notre deuil, et privatiser le système de protection sociale. Parmi les plus éminents défenseurs de 
cette thèse figurent les professeurs Meinhard Miegel, président de l'Institut für Wirtschaft und Gesellschaft (IWG, Bonn), et Kurt Biedenkopf. Ce dernier, l'un des pères de la cogestion, fut ministre-président de la Saxe avant de se voir confier par le gouvernement Schröder la présidence de la Commission chargée d'élaborer une réforme de la cogestion. Tous deux ne cessent, depuis les années 1970, d'invoquer surtout l'impact des mutations démographiques pour fonder leur critique à l'encontre d'un Etat Providence hypertrophié et d'une culture d'assistance qui n'est plus finançable aujourd'hui. Ils prônent une baisse des prélèvements pour permettre aux salariés de s'assurer par eux-mêmes. Dans ce même groupe, d'autres voix encore s'élèvent, qui considèrent plutôt le vieillissement comme source de conflits inter-générationnels.

La seconde approche est celle d'un groupe qu'on pourrait appeler les "démythificateurs » étant donné qu'ils dénient tout caractère dramatique aux mutations démographiques. Ils réfutent la thèse selon laquelle le vieillissement nécessiterait une réforme du système de protection sociale. C'est plutôt le haut niveau de chômage actuel et la relative stagnation des revenus réels qui menacent, selon eux, l'avenir du financement de l'Etat Providence. Dans leur discours, le facteur démographique n'est pas l'argument premier justifiant les réformes en cours, mais un simple prétexte pour réduire drastiquement les acquis sociaux. Dans cette approche, le vieillissement n'est donc considéré comme problématique que dans un seul cas : s'il mène, dans la société, à la prédominance d'un groupe profitant d'une redistribution du bas vers le haut. Cette doctrine est défendue par une partie des syndicats et de la gauche.

La troisième approche, celle que nous partageons ici, est celle des "organisateurs ». Elle défend la thèse que les mutations démographiques, dans la mesure où elles affectent l'organisation et le fonctionnement de la société dans son ensemble, doivent être accompagnées. Cet accompagnement des évolutions de l'ordre social constitue une mission collective, impartie à l'ensemble de la société. Or dans un pays qui se caractérise par une société organisée, cette mission d'accompagnement et d'organisation ne peut être que consensuelle, portée par tous, et elle requiert l'engagement responsable de tous les groupes représentatifs de la société. Les tenants de cette approche défendent une position médiane. Ils se recrutent dans la majorité des partis élus au Bundestag et des courants représentés dans les syndicats. Leur discours est critique puisqu'il affronte les défis ; mais il est aussi et avant tout optimiste. Car le vieillissement et la baisse de la population sont perçus comme une opportunité : ils forcent à considérer les choses sous un angle nouveau, générant ainsi des idées novatrices permettant d'aménager l'avenir dans une perspective de progrès.

\section{Vieillissement démographique et Etat social}

Le système allemand de protection sociale reposant largement sur le travail salarié, son financement est tributaire du nombre de cotisants. Or actuellement, le marché de l'emploi se caractérise par un taux d'activité moyen, un niveau de chômage élevé et un recul du nombre d'emplois soumis à cotisations sociales, ce qui se traduit par une baisse dramatique des recettes du système de protection sociale dont le déficit s'accentuera ainsi sous l'effet du vieillissement.

Car à l'avenir, le nombre de cotisants va diminuer et celui des bénéficiaires des prestations, augmenter. En 2050, l'Allemagne ne comptera plus qu'un peu moins de 40 millions de personnes en âge de travailler au lieu de quelque 50 millions aujourd'hui (Destatis, 2003). Or le vieillissement affectera également le volume comme la structure des dépenses des systèmes de protection sociale. La hausse du nombre de personnes âgées et l'augmentation de l'espérance de vie accroîtront dans le même temps les dépenses par tête pour les prestations.

Mais ces effets n'affecteront pas de la même manière les régimes de protection retraite, maladie ou dépendance. C'est dans le cas des retraites que l'impact du
Ceux qui « démythifient »

Ceux qui accompagnent et « organisent "

Une protection sociale trop centrée sur le travail salarié

A moyen terme, baise des recettes et hausse des dépenses

Impact direct du vieillissement sur le financement des retraites 
Santé : risque de morbidité accru ou au contraire réduit?

Impact direct du vieillissement sur le risque dépendance

Facteur aggravant : le faible taux d'activité des seniors

1992-2002 : Commission d'enquête «Mutations démographiques"

Le gouvernement Merkel lance la retraite à 67 ans vieillissement est le plus évident: l'accroissement des dépenses est directement lié à la fois à l'augmentation du nombre de retraités et à l'allongement de la durée de versement des pensions (Fasshauer, 2005).

La hausse des dépenses de santé s'explique par les progrès de la médecine, auxquels s'ajoutent les effets spécifiques du vieillissement que sont un risque de morbidité accru et, parallèlement, une consommation renforcée de prestations médicales. C'est là la thèse de la médicalisation défendue par une partie de la communauté scientifique. D'autres chercheurs, à l'opposé, défendent la thèse d'une compression de la morbidité. Elle prévoit que les dépenses de santé ne devraient pas croître de manière significative puisque les assurés seront en meilleure santé jusqu'à un âge avancé et que, en conséquence, les prestations au coût élevé se concentreront sur la brève période précédant leur mort (Jacobs/Dräther, 2005). Ces prévisions se laissant toutes deux étayer par les résultats d'études empiriques, les effets prévisionnels du vieillissement sur l'assurance maladie se situeront probablement entre ces deux scénarios.

Quant à la hausse des dépenses de l'assurance dépendance, elle résulte de l'augmentation de personnes très âgées, un groupe présentant un risque élevé de dépendance. Si $10 \%$ seulement des 75-80 ans sont en situation de dépendance, cette part passe à $35 \%$ chez les $80-90$ ans et même à $52 \%$ chez les plus de 90 ans (Rothgang, 2005).

A ces défis liés à l'évolution démographie s'ajoutent des dysfonctionnements majeurs du marché de l'emploi. En Allemagne, le taux d'activité des seniors est plus faible que dans la plupart des Etats membres aux économies compétitives. II n'est que de $39 \%$ chez les 55-64 ans. En France, ce taux est comparable aujourd'hui (39,9\% en 2003) grâce à une hausse de 5,9\% depuis 1994 (en Allemagne, elle n'a été que de $3 \%$ ). A l'opposé, deux pays se distinguent : le Danemark, avec un taux de $60,7 \%(+10,5 \%)$, et la Suède, avec $69 \%(+7,1 \%$; Allmendinger, 2005). En Allemagne, ce faible taux reflète une réalité préoccupante : dans plus de la moitié des entreprises, aucun salarié n'a plus de 50 ans. S'y ajoute la faible propension des entreprises à embaucher des seniors ou à les faire bénéficier de mesures de formation continue (Bellmann et al., 2003). On comprend mieux le caractère préoccupant de cette discrimination liée à l'âge dans une société vieillissante quand on sait que l'âge moyen des personnes en âge de travailler passera de 41 ans aujourd'hui à 48 ans en 2050.

\section{Comment le gouvernement fédéral relève le défi}

Le gouvernement fédéral n'a relevé que très tard le défi du vieillissement. Pourtant, les voix n'avaient pas manqué pour insister sur la nécessité d'agir. C'est en octobre 1992 seulement que le gouvernement Kohl institua une Commission d'enquête «Mutations démographiques - les défis que pose notre société vieillissante aux citoyens comme au monde politique ». Ces Commissions (Enquetekommission), inscrites dans la durée puisque leurs travaux s'étendent sur plusieurs législatures, se composent de députés du Bundestag (majorité et opposition) et de scientifiques ; à l'issue de leurs travaux, elles publient un rapport comprenant une analyse exhaustive de la problématique et un catalogue différencié de propositions (plusieurs alternatives) d'action à l'attention des acteurs politiques. La commission sur la démographie siégea dix ans. Mais ses propositions restèrent en grande partie lettre morte.

Parmi les rares propositions retenues figure la hausse de l'âge légal de départ à la retraite ; il est vrai qu'elle est prônée par de nombreux experts. Le gouvernement Merkel projette ainsi de faire adopter la réforme à l'automne. Dès 2012, l'âge légal de départ à la retraite passera progressivement de 65 à 67 ans, seuil qui sera atteint en 2019. Néanmoins, au-delà de cette date, les salariés pourront continuer à bénéficier d'une retraite de plein droit dès l'âge de 65 ans s'ils 
ont cotisé pendant 45 annuités ; l'objectif de cette mesure est de maintenir en emploi les seniors et de permettre aux chômeurs âgés de retrouver un emploi.

Actuellement, l'âge moyen de départ à la retraite est d'environ 60 ans, ce qui se traduirait par un taux de vieillesse de $77,8 \%$ en 2050 . Si cet âge moyen correspondait à l'âge légal actuel (65 ans), ce taux ne serait plus que de $54,5 \%$; il tomberait même à 47,4 \% pour un âge légal porté à 67 ans (Destatis, 2003). Une chose est certaine : l'âge effectif de départ à la retraite va augmenter au cours des prochaines années étant donné que l'ensemble du dispositif d'incitation à la retraite anticipée sera supprimé d'ici 2009. Parallèlement, le gouvernement avait commencé dès 2002 à mener une politique de soutien au développement du système des retraites d'entreprise (voir REA 70/05) - une politique expressément saluée et accompagnée par les partenaires sociaux.

Pour remédier au faible taux d'activité des seniors, le gouvernement fédéral a lancé en juillet 2006 le programme Initiative 50 plus. Dans ce cadre, il projette de développer un modèle de subventions salariales (Kombilohn) comprenant des aides à la réinsertion professionnelle des seniors et un régime spécifique de formation continue. Ce programme, financé à hauteur de 250 millions $€$, comprend plus précisément les mesures suivantes:

- l'entreprise qui embauche un salarié de plus de 50 ans bénéficie pendant deux ans au maximum d'une subvention de réinsertion d'un montant compris entre $20 \%$ et $40 \%$ du salaire brut, à condition que le contrat de travail soit d'une durée d'un an au minimum ;

- les plus de 50 ans bénéficiaires de l'assurance chômage (Arbeitslosengeld I) qui reprennent un emploi dont la rémunération est nettement inférieure au salaire perçu avant l'entrée en chômage perçoivent une indemnité compensatoire de 50 \% de l'écart (salaires nets) la première année et de $30 \%$ la seconde. Leurs cotisations retraite sont prises en charge jusqu'à hauteur de $90 \%$;

- dans les entreprises de moins de 250 salariés, le coût des mesures de formation continue des salariés de plus de 45 ans est partiellement pris en charge par l'Agence fédérale pour l'emploi ;

- le régime des contrats de travail à durée déterminée des plus de 50 ans est mis en conformité avec le droit européen (limitation à 4 ans de la durée des CDD).

Le programme gouvernemental Intitiative 50 plus s'inscrit dans le prolongement de l'Initiative neue Qualität der Arbeit (Initiative INQA), un programme lancé conjointement en 2002 par les partenaires sociaux, les caisses d'assurance sociale, le Bund, les Länder, des fondations et diverses entreprises dans l'objectif de développer l'aptitude au travail des seniors afin de préserver l'innovation et la compétitivité futures d'une Allemagne aux salariés vieillissants. Les mesures de ce programme sont toutes orientées sur l'efficience du transfert des savoirs grâce à la mise en réseau des savoir-faire des entreprises en matière de sécurité et de santé et, sur cette base, au développement de préconisations concrètes pour la pratique des entreprises.

Le programme Initiative 50 plus du gouvernement fédéral vise, lui, à promouvoir l'allongement de la durée de la vie professionnelle. En le lançant, le gouvernement a mis l'accent sur la nécessité de prendre des dispositions inhabituelles pour accompagner les mutations. Mais les ressources prévues sont trop faibles pour permettre de relever véritablement le défi de l'emploi des seniors. Si, en ce sens, l'Initiative se révèle une goutte d'eau dans la mer, on peut néanmoins en espérer un effet stimulant sur la motivation des entreprises et des partenaires sociaux à reconsidérer l'activité des plus de 50 ans.

En matière de politique familiale, l'Allemagne s'est engagée dans une nouvelle voie pour permettre de mieux concilier vies familiale et professionnelle. C'est ainsi qu'une nouvelle allocation parentale est versée au père ou à la mère qui se consacre à l'éducation de l'enfant pendant sa première année. Cette allocation, plafonnée à $1800 €$ par mois et versée pendant 12 mois, représente $67 \%$ du dernier salaire net perçu. Par ailleurs, le gouvernement fédéral avait lancé dès 2005 un programme visant à garantir une structure d'accueil à tous
Depuis 2002, développement des retraites d'entreprise

Juillet 2006 : lancement du programme Initiative 50 plus

II prolonge le programme collectif Initiative INQA lancé en $2002 .$.

... et vise l'allongement de la durée de la vie professionnelle

Une politique pour concilier vies familiale et professionnelle 
Des lieux pour une nouvelle solidarité intergénérationnelle les enfants de moins de 3 ans. Certes, personne n'ignore que le taux de natalité ne peut progresser que très lentement étant donné la multitude de facteurs en jeu, mais ces deux mesures gouvernementales n'en constituent pas moins des signaux forts dont on espère qu'ils influenceront la natalité. Cette politique répond en tout cas aux préoccupations des Allemands. Dans un récent sondage, $66,3 \%$ des personnes interrogées, âgées de 20 à 45 ans, laissent entendre que si leurs conditions de vie et de travail s'amélioraient, elles planifieraient autrement leur vie familiale (Dorbritz et al., 2005).

En outre, le gouvernement fédéral soutient la création de « maisons plurigénérationnelles » (Mehrgenerationenhäuser), conçues comme des lieux d'accueil et d'échanges offrant toute la gamme des prestations liées à la famille : conseil en éducation, planification familiale, santé, gestion des crises et aides diverses. L'idée est de recréer des liens comme ceux qui unissaient les familles multigénérationnelles d'autrefois, mais au sein de structures externes, ouvertes la journée et accueillant toutes les générations dans la même optique d'entraide. C'est là une approche qui, tout en reconnaissant la réalité qu'est aujourd'hui la prédominance des familles cellulaires, tente d'offrir la possibilité de renouer les liens de solidarité entre les âges.

Ces Politiques, qu'elles concernent l'âge de la retraite, les transferts sociaux ou le développement des infrastructures d'accueil, ne sont encore que de timides mesures, mais elles cherchent toutes à mieux prendre en considération désormais les besoins d'une société vieillissante. II n'en reste pas moins que les gouvernements allemands se sont préoccupés trop tard d'accompagner par l'action les mutations démographiques. Certes, les instruments et champs d'action politiques ne manquent pas pour en accompagner les effets et aménager l'avenir : une réforme des systèmes de protection sociale s'impose pour préserver son financement comme sa qualité ; des mesures spécifiques aux salariés vieillissants sont inévitables pour garantir la capacité d'innovation des entreprises ; une autre politique familiale s'impose pour mieux concilier vies familiale et professionnelle. Mais il est une tendance qui se révèle hautement problématique : c'est la propension à justifier les réformes structurelles incontournables par le seul et vague argument d'un vieillissement global de la population. Car elle nourrit le soupçon : cette politique ne servirait-elle pas en réalité à camoufler des conflits de répartition? Cela handicape toute politique qui chercherait à répondre avec le doigté requis aux réalités hautement complexes que recouvrent les mutations démographiques. Les quelques mesures adoptées jusqu'ici sont tout au plus un début. Le « modèle allemand » est encore loin d'être armé pour affronter le risque démographique.

Traduction : I.B.

\section{Indications bibliographiques}

ALLmendinger J. et al., IAB Handbuch Arbeitsmarkt, Francfort, 2003

BELLMANN L. et al., " Herausforderungen des demographischen Wandels für den Arbeitsmarkt und die Betriebe ", in Mitteilungen aus der Arbeitsmarkt- und Berufsforschung, $2 / 2003$

DORBRITZ J. et al., Einstellungen zu demographischen Trends und zu bevölkerungsrelevanten Politiken, Wiesbaden, 2005

FASSHAUER S., "Die Folgen des demographischen Wandels für die gesetzliche Rentenversicherung ", in KeRSCHBAUMER J., SCHROEDER W. (eds), Sozialstaat und demographischer Wandel. Herausforderungen für Arbeitsmarkt und Sozialversicherung, Wiesbaden, 2005

Jacobs K ., DräHTER H., « Die Folgen des demographischen Wandels für die gesetzliche Krankenversicherung ", in KERSCHBAUMER J., SCHROEDER W. (eds), op.cit.

MıCHEEL F., « Die demographische Entwicklung in Deutschland und ihre Implikationen für Wirtschaft und Soziales ", in Kerschbaumer J., SCHROEder W. (eds), op.cit.

MAI R., Die Alten der Zukunft. Eine bevölkerungsstatistische Datenanalyse, Opladen, 2003

Rothgang H., "Demographischer Wandel und Pflege(ver)sicherung ", in KerschBaUMER J., SCHROEDER W. (eds), op.cit.

Statistisches Bundesamt (Destatis), Bevölkerung Deutschlands bis 2050. 10. koordinierte Bevölkerungsvorausberechnung, Wiesbaden, 2003. 